\title{
Myasthenia Gravis Presenting Like Guillain-Barré Syndrome
}

\author{
Isha Misra $^{a}$ Frehiwot D. Temesgen $^{a} \quad$ Noha Soleiman $^{a}$ \\ Janaki Kalyanam $^{\mathrm{b}}$ Mohankumar Kurukumbi ${ }^{\mathrm{a}}$ \\ ${ }^{a}$ Department of Neurology, Howard University Hospital, and ${ }^{b}$ Department of \\ Physical Medicine and Rehabilitation, Howard University Hospital, \\ Washington, D.C., USA
}

\section{Key Words}

Myasthenia gravis · Guillain-Barré syndrome · Diabetes mellitus

\begin{abstract}
Myasthenia gravis (MG) is an autoimmune disorder characterized by weakness in specific muscle groups, especially the ocular and bulbar muscles. Guillain-Barré syndrome (GBS) presents with ascending paralysis and areflexia, often secondary to an infection. Several theories have been proposed regarding the etiology behind GBS, with many studies pointing to a possible autoimmune cause. If this is in fact true, it is also possible that the two diseases may develop concurrently. While this is unusual, several recently published studies highlight such cases of concurrent MG and GBS. This co-occurrence could involve certain common proteins, as the two diseases can present somewhat similarly. This is an unusual case of a patient with no significant past medical history, presenting with generalized weakness and symptoms of new-onset diabetes, who developed bilateral ptosis, distal weakness, and areflexia while in the hospital, raising the possibility of concurrent MG and GBS. Although the diagnosis of MG was confirmed by the positive anticholinesterase antibodies and tensilon test, several features, including sudden onset of ascending paralysis and areflexia, were more common in GBS than MG. It is possible, albeit rare, that these two syndromes could have developed concurrently and that the untreated diabetes mellitus could have contributed to the neurological symptoms. This case is reported because of the rarity of its features, diagnostic and management challenges.
\end{abstract}

\section{Introduction}

Myasthenia gravis (MG) is an autoimmune disorder involving development of autoantibodies against specific proteins in the postsynaptic membrane of the neuromuscular junction, typically against the acetylcholine receptor (AChR) or related proteins. It is characterized by weakness, often in the ocular, bulbar, limb, and 
respiratory muscle groups. This disorder is often insidious and, as a result, may go undiagnosed for a significant period of time until an acute exacerbation occurs [1]. While it is well known that the mechanism behind MG is autoimmune, there are several proposed etiologies for Guillain-Barré syndrome (GBS), which is also known as acute immune-mediated polyneuropathy. GBS involves ascending muscle weakness. It is usually symmetric and is accompanied by areflexia. Patients must be closely monitored, as they can quickly develop respiratory failure [2].

There is significant evidence supporting a possible autoimmune etiology for GBS [3] If this is in fact true, it is also possible that the two diseases may develop concurrently. While this is unusual, several recently published studies highlight such cases of concurrent MG and GBS. This co-occurrence could involve certain common proteins, as the two diseases can present somewhat similarly.

\section{Case Report}

A 36-year-old male patient with no significant past medical history presented to the emergency room with symptoms of generalized weakness, dehydration, and distal weakness in both upper and lower extremities for 2 weeks. Upon physical examination, the patient was tachycardic and appeared lethargic. His fingerstick was recorded as $305 \mathrm{mg} / \mathrm{dl}$ (normal values: 70-99 mg/dl), and his urinalysis was significant for a high urine glucose, large ketones, and trace protein. The patient was determined to be hyperglycemic, likely due to new-onset diabetes mellitus, and was admitted to the hospital for further management of his symptoms.

Over the next few days, the patient developed bilateral ptosis and his distal weakness persisted, involving bilateral wrist and foot drop. Extra-ocular movements were impaired in all directions (fig. 1a), and he had an expressionless face due to involvement of facial muscles. The patient's tachycardia also persisted with his heart rate ranging from 110 to $130 \mathrm{bpm}$. Upon further examination, sensation was intact, but total areflexia was noted. The patient's weakness progressed, and shortly after, he began to complain of dysphagia and associated weakness upon attempted flexion of his head. Upon further questioning, the patient remembered experiencing a transient unilateral ptosis 2 weeks before admission, but denied experiencing any other myasthenic symptoms.

Based on these findings and the progression of the symptoms, two main diagnoses were considered: MG with acute exacerbation and GBS. Additional tests were then ordered so as to make a diagnosis in this vein. MRI of the brain was normal, and nerve conduction studies (NCSs) showed prolongation of $\mathrm{F}$ waves, decrease in compound muscle action potentials, and marked reduction in conduction velocities in bilateral tibial, peroneal, median, and ulnar nerves. This suggests a mixed pattern of both axonal and demyelinating features. There was no conduction block or temporal dispersion present. Additionally, repetitive nerve stimulation studies of abductor pollicis brevis were performed and resulted in a decremental response ( fig. $2_{2}$ ). The tensilon test was conducted and showed improvement in ophthalmoplegia. At this time, AChR antibody levels were found to be significantly elevated $(55.00 \mathrm{nmol} / \mathrm{l}$, with normal levels being $<0.30 \mathrm{nmol} / \mathrm{l}$ ) confirming the diagnosis of MG. A lumbar puncture was performed and revealed no white blood cells (normal values: $0-5 \mathrm{~mm}^{3}$ ) and a protein level of $95 \mathrm{mg} / \mathrm{dl}$ (normal values: $15-45 \mathrm{mg} / \mathrm{dl}$ ) suggesting albuminocytologic dissociation. As the patient's weakness progressed, he developed respiratory distress and was intubated. At this time, he was transferred from the neurology service to the medicine intensive care unit. The clinical features of areflexia, albuminocytologic dissociation, and ascending paralysis leading to respiratory failure were all suggestive of GBS. The Miller-Fisher variant of GBS was considered at this point due to the patient's ophthalmoplegia. Based on this, the GQ1b (anti-ganglioside Q1b) antibody test was performed and was found to be negative.

After the intubation, five cycles of plasmapheresis were completed, after which the patient was started on pyridostigmine. The patient was successfully weaned from the ventilator, but was later found to have increased secretions due to the pyridostigmine. As a result, he was re-intubated, and pyridostigmine was discontinued. At this time, MG was considered to be the main diagnosis. As 
plasmapheresis is known to only have a transient effect, the patient was also started on $40 \mathrm{mg}$ of prednisone. Since the patient's glycosylated hemoglobin levels were 14\% (normal values: 3.4-6.1\%), his blood glucose levels were optimized with insulin. Despite these treatments, it remained difficult to wean the patient off the respirator, and other factors for the failure to extubate were ruled out. At this point, five doses of intravenous immunoglobulins were given, and clinically, the patient began to improve, although his progress was slow. Mycophenolate was started as a steroid-sparing agent, and pyridostigmine was later restarted at low doses. The patient continued to improve and the deep tendon reflexes returned to normal in lower and upper extremities. With regard to his new-onset diabetes, the patient was found to have glutamic acid decarboxylase (GAD) antibodies. Eventually, the patient improved enough to be discharged to a specialty hospital for long-term care (fig. 3 ).

Written informed consent was obtained from the patient for publication of this case report and accompanying images.

\section{Discussion}

MG is an autoimmune disorder which arises due to an antibody-mediated T-celldependent attack on the proteins present in the postsynaptic membrane of the neuromuscular junction. Usually, these are antibodies against the nicotinic AChRs or receptor-associated proteins. Typically, patients who have MG present with a weakness in the ocular, bulbar, limb, and/or respiratory muscles. Some patients may only have ocular symptoms, while others may have more generalized symptoms [1].

The weakness in MG varies in severity and in location from person to person. Initially, the patient will experience a relapsing- and remitting-type pattern; but as the disease progresses, the symptoms persist, although they still may fluctuate in severity. In this case, the patient presented with ocular symptoms that are typical for MG bilateral ptosis and ophthalmoplegia. However, the symptoms did not fluctuate as seen in most cases. Our patient also had bilateral facial muscle weakness resulting in a myasthenic sneer, which is a rare clinical feature. On occasion, patients may present with a dropped head syndrome, as the neck extensor muscles give out, but interestingly, our patient had trouble with flexion, rather than extension of his head. Furthermore, the limb weakness experienced by MG patients is typically proximal, and the arms often appear to be affected more by the disease than the legs. Distal limb weakness (as seen in our patient) is less common, but has been noted in about $10 \%$ of cases in a few studies involving MG patients [4].

This case is also notable for the fact that the patient had no known past medical history of symptoms attributable to MG, and he presented with acute exacerbation subsequently involving the respiratory muscle weakness. Weakness involving respiratory muscles is rarely the presenting feature in the first 2 years of onset [5]. On occasion, the patient will present with respiratory insufficiency that is out of proportion to his limb or bulbar symptoms, as seen with our patient. This is a serious situation, and these patients should be admitted to the intensive care unit, where vital capacity and negative inspiratory force are the main parameters used to monitor their progress. There are many precipitants, but the most common cause is a concurrent infection. Other causes include surgery, pregnancy, childbirth, or tapering of immunosuppressants. Additionally, acute exacerbation can be secondary to certain medications (aminoglycosides, $\beta$-blockers, and magnesium). All these precipitating factors were excluded in our patient. 
As discussed above, we also tested for the presence of GAD antibodies in this patient based on his initial presentation with symptoms of new-onset diabetes mellitus. These antibodies have been classically associated with stiff-person syndrome; however, their presence simply suggests that the patient's symptoms are attributable to an autoimmune disease [6]. GAD has been shown to be involved in the synthesis of GABA, and antibodies directed against GAD are present in several other autoimmune diseases, including diabetes mellitus and MG [7].

Although MG was confirmed in this patient, the presence of ascending paralysis, areflexia, persistent tachycardia, prolonged $\mathrm{F}$ waves, demyelinating results of the NCSs, and albuminocytological dissociation in cerebrospinal fluid were all suggestive of a possible concurrent diagnosis of GBS. The NCS indicated an axonal loss and demyelination, which can be seen with GBS and also with polyneuropathy secondary to complications of diabetes mellitus.

GBS is characterized by a progressive and symmetric muscle weakness, accompanied by absent or decreased deep tendon reflexes, as seen with this patient. Typically, the weakness in GBS begins in the legs, although some patients have experienced initial arm and/or facial muscle weakness. Ptosis and other oculomotor signs have also been seen on occasion [2]. Additionally, patients may experience considerable respiratory muscle weakness and may subsequently need to be intubated. Paresthesias, along with back and limb pain, are often experienced especially in the acute stages; and autonomic dysfunction may be present. These are, however, nonspecific symptoms and can be seen in other diseases, such as diabetes mellitus.

GBS is often associated with a respiratory or gastrointestinal infection. There are various triggers or precipitants, including viral illnesses, surgery and vaccinations. None such triggers were found in our patient. There are several theories regarding the exact etiology behind GBS, but the prevailing one suggests an autoimmune mechanism. One popular theory is that of molecular mimicry, in which the body responds to certain antigens (from the infectious agent, vaccine, etc.) which have a similar composition to host proteins, and an attack is thus launched against the host [3]. Patients with GBS can present with a variety of symptoms. Thus, it is often helpful to look at the diagnostic criteria provided by the National Institute of Neurological Disorders and Stroke, which are as follows: progressive weakness of more than one limb (also trunk, bulbar, and facial muscles, and/or external ophthalmoplegia) and areflexia. Supportive features include progression of symptoms, relative symmetry of symptoms, cranial nerve involvement, autonomic features, elevated protein with cell counts, afebrile, electromyogram and nerve conduction abnormalities consistent with GBS, mild sensory deficits, and lastly a halt in the progression of the disease and a clear recovery [3]. Note that albuminocytologic dissociation is not listed in the above criteria.

Albuminocytologic dissociation is a nonspecific finding seen in the cerebrospinal fluid [8]. Typically, the results of NCSs in GBS are suggestive of demyelination, such as a delay in $\mathrm{F}$ waves, decreased nerve motor action potentials, and/or conduction block. The abnormalities must be seen in at least two nerves, which are rarely affected in other types of neuropathies, to be consistent with GBS [9].

As these criteria are quite broad, some of our patient's symptoms that do not fit the primary diagnosis of MG can then be considered to be a result of GBS. However, additional testing would be necessary in order to confirm this. The patient had some 
symptoms that were very atypical for MG. For instance, the ocular symptoms are normally more prominent and distal weakness is rarer in MG patients. A few studies have shown this co-occurrence. For example, in 2008, Lau et al. [10] described the case of an 84-year-old Chinese woman with a co-occurrence of MG and GBS (Miller-Fisher variant). The investigators proposed that since the patient's immune system had already been activated due to the underlying MG, it increased the likelihood that autoantibodies against other antigens could develop in the same set of muscles. Unlike our patient, however, this woman had already been diagnosed with MG for some time prior to her admission [10]. Another study published the following year discussed a similar case and proposed that the concept of molecular mimicry was responsible for the co-occurrence. This case, described by Kung et al. [11], was similar to ours in that the patient had no known history of symptoms that could be attributed to MG. A third article by Helgeland et al. [12], published in 2010, again supports the theory of antibodies, in this case, against heat shock proteins. Here, the researchers compared antibody levels in patients with MG, GBS, multiple sclerosis, and healthy controls. They concluded that the MG patients had higher antibody levels than the patients with MS and the healthy controls. The patients with GBS had the highest antibody levels of all the groups [12].

It is also possible that the unusual symptoms can be attributed to diabetes mellitus. Watkins and Thomas [13] found that while diabetic sensory polyneuropathy is the most common neurological manifestation, other symptoms include acute onset painful neuropathy, which presents with burning pain and hyporeflexia, and autonomic neuropathy, which causes sympathetic denervation.

Management of MG and GBS patients involves plasmapheresis or intravenous immunoglobulin. Both therapies have been found to be equally effective; yet, plasmapheresis is preferred due to a more rapid onset of action. These treatments have been shown to only be a temporary solution, however, and it is therefore important to also initiate high-dose steroids for long-term treatment in MG patients. However, longterm immunomodulation treatment has not been shown to be necessary in patients with GBS Miller-Fisher variant.

In summary, this patient had a confirmed diagnosis of MG with additional interesting atypical features or a possible co-occurrence of GBS. Furthermore, some of these neurological features were possibly contributed by undiagnosed diabetes mellitus. The rarity of this co-occurrence is intriguing, and extensive review of the literature showed only two such cases of MG co-existing with GBS. This case is a unique and rare occurrence, and additional research needs to be conducted to understand the mechanism behind this co-occurrence. There is evidence supporting the production of certain antibodies in both MG and GBS, and it would be therefore interesting to take a closer look at the similarities in how these two diseases arise.

\section{Disclosure Statement}

The authors declare that they have no competing interests. 


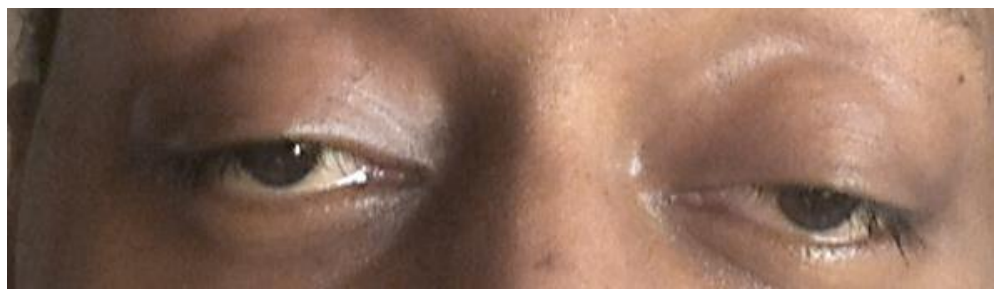

Fig. 1. Photograph of patient exhibiting bilateral ptosis and ophthalmoplegia at the time of admission.
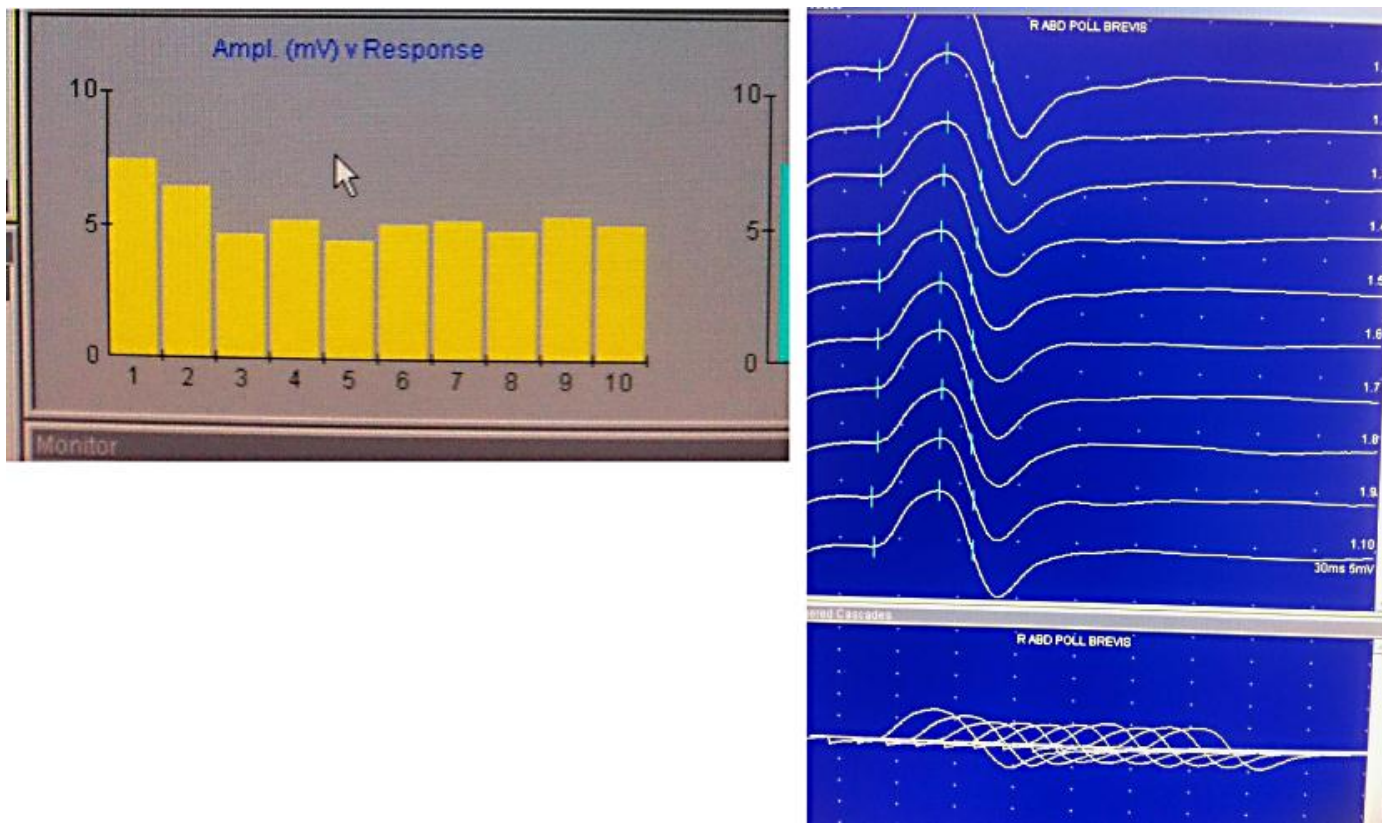

Fig. 2. This is a photograph showing decremental response to repetitive nerve stimulation of right abductor pollicis brevis.

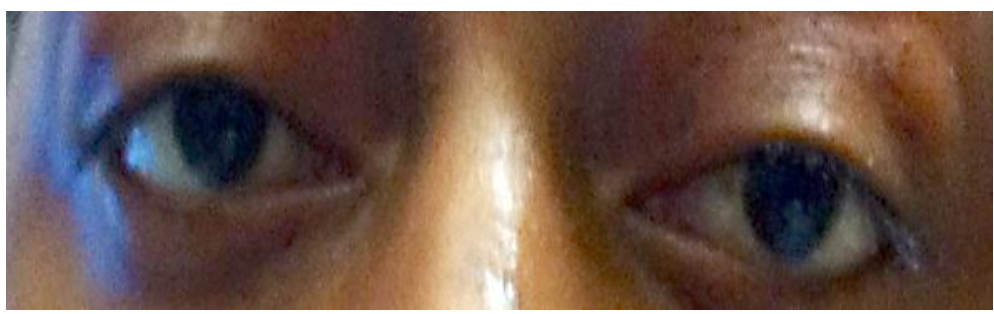

Fig. 3. Photograph showing improvement in the patient's ocular symptoms at the time of discharge. 


\section{References}

1 Drachman DB: Myasthenia gravis. N Engl J Med 1994;330:1797-1810.

2 Teng HW, Sung JY: Ptosis as the initial presentation of Guillain-Barré syndrome. J Emerg Med 2010, Epub ahead of print.

3 Drachman DB: Myasthenia gravis and other diseases of the neuromuscular junction; in Longo DL, Fauci AS, Kasper DL, Hauser SL, Jameson JL, Loscalzo J (eds): Harrison's Principles of Internal Medicine, ed 18. New York, McGraw-Hill, 2012. http://www.accessmedicine.com/content.aspx?aID=9148895 (accessed April 22, 2012).

-4 Nations SP, Wolfe GI, Amato AA, Jackson CE, Bryan WW, Barohn RJ: Distal myasthenia gravis. Neurology 1999;52:632-634

5 Grob D, Brunner N, Namba T, Pagala M: Lifetime course of myasthenia gravis. Muscle Nerve 2008;37:141-149.

6 Thomas S, Critchley P, Lawden M, Farooq S, Thomas A, Proudlock F, Constantinescu C, Gottlob I: Stiff person syndrome with eye movement abnormality, myasthenia gravis, and thymoma. J Neurol Neurosurg Psychiatry 2005;76:141-142.

7 Batstra MR, van Driel A, Petersen JS, van Donselaar CV, van Tol MJ, Bruining GJ, Grobbee DE, Dyrberg T, Aanstoot HJ: Glutamic acid decarboxylase antibodies in screening for autoimmune diabetes: influence of comorbidity, age, and sex on specificity and threshold values. Clin Chem 1999;45:2269-2272.

$>8$ Ropper AH, Marmarou A: Mechanism of pseudotumor in Guillain-Barré syndrome. Arch Neurol 1984;41:259.

9 National Institute of Neurological Disorders and Stroke: Guillain-Barre syndrome. http://www.ninds.nih.gov/disorders/gbs/detail_gbs.htm?css =print (accessed April 22, 2012).

10 Lau KK, Goh KJ, Lee HC, Chan YT, Tan CT: The co-occurrence of serologically proven myasthenia gravis and Miller Fisher/Guillain Barré overlap syndrome: a case report. J Neurol Sci 2009;276:187-188.

11 Kung SL, Su JM, Tsai SJ, Lu TM, Chen CM: Concurrent Guillain-Barré syndrome and myasthenia gravis: the first case in Taiwan. Acta Neurol Taiwan 2009;18:193-197.

12 Helgeland G, Petzold A, Hoff JM, Gilhus NE, Plant GT, Romi FR: Anti-heat shock protein 70 antibody levels are increased in myasthenia gravis and Guillain-Barré syndrome. J Neuroimmunol 2010;225:180-183.

13 Watkins P, Thomas P: Diabetes mellitus and the nervous system. J Neurol Neurosurg Psychiatry 1998;65:620-632. 